\title{
La mise au carré du langage et de la pensée: une technique au service du recadrage en intervention systémique
}

\author{
Daniel Faulx Psychologue, Professeur, Université de Liège, ARCH \\ Coryse Moncarey Psychologue, Université Catholique de Louvain
}

\section{Résumé}

Le recadrage constitue une démarche centrale dans la thérapie systémique et notamment dans les thérapies narratives et orientées solution. Dans cet article, nous présentons une technique particulière dénommée mise au carré. Nous en envisageons le déploiement au service du recadrage dans un contexte d'intervention systémique. Fondée sur le repérage des "liaisons", la mise au carré permet de visualiser les associations existantes dans un discours et d'explorer celles qui sont en germe mais non exprimées par la personne. Elle vise le déploiement de nouvelles associations potentielles au service de l'évolution du client. L'article présente une étude de cas qui vient illustrer la démarche. Les implications pour la pratique thérapeutique sont discutées en fin d'article.

\section{Introduction}

Dans cet article, nous exposons une technique particulière pouvant soutenir la création de recadrage dans le contexte d'intervention en thérapie systémique. Dénommée mise au carré (Faulx et Danse, 2017), cette technique a pour but de faciliter l'analyse d'une pensée et l'émergence de pistes alternatives. Son apport est d'aider à l'exploration avec le client d'idées en germe dans son discours mais non encore envisagées jusque-là (Fossion, Jamin et Faulx, 2018).

Au travers d'une étude de cas, nous examinerons de quelle manière l'utiliser en appui de l'intervention systémique. 


\section{Ouvrir le champ des possibles, une clé de voûte de lintervention systémique}

Aider quelqu'un à modifier son point de vue sur une situation, à voir un problème sous un angle nouveau, à appréhender le monde d'une manière inédite, en somme l'aider à multiplier les points de vue afin de se donner la possibilité de voir une situation autrement (Bandler et Grinder, 2014) constitue une démarche essentielle dans la pratique systémique.

Une telle opération de décentration est mobilisée dans des contextes très variés d'intervention: en organisation (Malarewicz, 2000), en milieu scolaire (Curonici et McCulloch, 2004), dans le traitement des hyperconflits familiaux ou professionnels (Faulx, 2006), en soins psychiatriques (Panichelli, 2007), dans l'accompagnement professionnel (Paul, 2009) ou encore dans le travail social où elle constitue la dernière phase (avant conclusion) du processus en sept étapes des techniques systémiques d'entretien proposé par Hanot (2006).

Soutenir un client, une famille ou une équipe dans sa capacité à développer de nouveaux regards s'apparente le plus souvent à la démarche dite de recadrage. Celle-ci peut se définir comme "tout ce qui modifie pour un patient ou une famille son modèle de représentation de la réalité" (Benoît, Malarewicz, Beaujean, Colas et Kannas, 1988). Thérapeute et patient partent alors ensemble à la découverte de nouvelles significations, d'autres manières de voir la situation-problème ou encore de récits alternatifs.

Ce type de démarche est très présent dans différentes approches de psychothérapie d'orientation systémique. Ainsi, dans les thérapies narratives, on considère que la réalité d'une personne est le fruit d'une construction sociale, souvent influencée par le discours social dominant (Gergen et White). Ce processus la conduit à étiqueter et catégoriser ses expériences de vie d'une certaine manière. L'enjeu est alors, avec la personne, de déconstruire les problèmes ou, pour mieux dire, de codéconstruire et coreconstruire son histoire afin qu'elle puisse la regarder autrement (Mori et Rouan, 2011).

Dans le même ordre d'idées, dans les thérapies orientées solution, étant donné que l'on travaille sur les changements et les solutions et non sur le problème du patient, la focale est mise sur des éléments que les personnes ont tendance à ne pas repérer ou négliger: ce qui marche déjà, les cas particuliers, les ressources présentes (de Shazer et Dolan, 2007; Doutrelugne et al., 2019; Petit, 2005). Travailler sur ces éléments permet à la personne de raconter une autre histoire et d'adopter des comportements plus constructifs (D'Abate, 2016). Même si on reconnaît les effets du passé, ce sont les affects, les pensées et les ressentis dans l'ici et le maintenant qui sont au centre de la thérapie. Comme l'indique l'auteur, dans les récits du patient, les personnages et les lieux sont identiques, par contre, la trame du récit, elle, change.

À partir de la narration de la personne, il s'agit donc de travailler sur les prémisses qui sont à la base de ses raisonnements et de ses comportements dans le but de les faire évoluer (Wittezaele et García, 1998). Robin-Quach (2009) 
insiste sur l'importance pour l'intervenant de s'employer d'abord à connaître les représentations (de la personne ou du système concerné) afin ensuite de pouvoir les faire évoluer. Cette double démarche (connaître - faire évoluer) est essentielle dans tout recadrage puisque cette entreprise ne peut fonctionner qu'à condition de prendre en compte la vision du monde exprimée par la personne ou le système concerné (Kourilsky-Belliard, 1995) avant d'envisager de la modifier.

De manière étonnante, tandis que le recadrage semble bien constituer la voie typique pour réaliser ce changement de point de vue essentiel à l'évolution du système, ce type de pratique, véritable "clé de voûte" pour l'intervention, ne fait l'objet que de rares publications dans la littérature (Janne et Dessoy, 1999).

Cela ne veut pas dire qu'il n'existe pas de suggestions pratiques dans la littérature. On sait par exemple que le recadrage peut être utilisé de manière uniquement verbale ou passer par des actions qui amènent la personne à changer son point de vue (Nardone et Watzlawick, 1993).

On note aussi qu'il peut correspondre à une intervention ponctuelle ou au contraire être déployé au fil des séances (Janne et Dessoy, 1999). Dans ce deuxième cas, il s'agit davantage d'un cheminement effectué à l'aide de questions que d'une intervention unique du thérapeute (Faulx, 2006). Dans cette optique, il ne suffit pas de faire un seul recadrage spectaculaire. Au contraire, généralement, plusieurs recadrages vont être nécessaires pour modifier petit à petit la vision du monde du client (Beaumont, 2016).

De son côté, Haley (1995) reprend différents procédés inscrits dans la démarche de recadrage: encourager la résistance, prescrire la rechute, présenter une option plus mauvaise, favoriser une action en s'y opposant, utiliser des métaphores et des analogies. Ces pistes pour la pratique sont construites autour de la notion de confusion chère à Erickson (demander au patient de "résister au thérapeute", prescrire des comportements spontanés, utiliser des alternatives illusoires ou formuler des questions paradoxales). La retrouver dans ce contexte n'est évidemment pas fortuit. En effet, le recadrage fait partie intégrante de la pratique de l'hypnose (Benoît, 1999).

On trouve aussi dans la littérature différentes propositions de pratiques comme:

- la question miracle/magique, considérée par certains comme un pilier de la thérapie (D’Abate, 2016; de Shazer et Dolan, 2007; Miller et de Shazer, 2001 ; Petit, 2005). Elle permet de "trouver des solutions pour construire une nouvelle réalité" (Petit, 2005) et aide le patient à se fixer des objectifs de changement atteignables;

- l'accent sur les exceptions aux problèmes (D’Abate, 2016), "voie royale vers les solutions", en ce sens qu'elles montrent au patient qu'il est capable d'exercer un contrôle sur sa situation (Isebaert et Cabié, 2015);

- les échelles de mesure (de Shazer et Dolan, 2007) qui aident à objectiver le ressenti du patient et à se voir évoluer de séance en séance. Il s'agit d'une lecture ponctuelle et chiffrée de sa perception de la réalité qui évolue par microchangements, parfois tellement petits qu'ils sont presque imperceptibles. 
Enfin, d'autres approches partagent ce même but d'ouvrir à d'autres visions de la réalité. Même si elles ne portent pas le nom de recadrage, elles permettent aussi de viser le changement de paradigme chez le patient (Marsan, 2008): passer par l'humour afin d'amener à d'autres interprétations de situations cliniques (Panichelli, 2007), utiliser des objets flottants pour ouvrir l'espace des possibles (Sprocq-Demarcq et Rey, 2008), ou encore recourir à des métaphores et des techniques non verbales pour neutraliser le pouvoir des mots (Goldbeter-Merinfeld, 2012).

\section{La mise au carré: une méthode d'intervention Principe général}

La mise au carré, qui va aider l'intervenant à développer ses recadrages, sert deux intentions complémentaires. D'une part, elle supporte la démarche de compréhension de l'intervenant et, d'autre part, elle stimule sa créativité pour dénicher, avec l'accompagné, de nouvelles visions de sa situation. On retrouve ici les deux volets de la démarche de Robin-Quach (2009): connaître et faire évoluer.

Le fondement de la démarche part du constat selon lequel la pensée d'une personne est caractérisée par une multitude de liaisons, souvent implicites (Faulx et Danse, 2017). Techniquement, une mise au carré commence donc par l'observation d'une liaison entre deux idées présentes dans le discours du client.

Les liaisons constituent alors la porte d'entrée pour identifier la manière dont pense une personne (versant analytique) et lui offrir d'autres modes de pensées possibles (versant créatif ou heuristique). Dans le premier temps, il s'agira de mettre en évidence les liaisons qui s'expriment dans son discours. Dans le second, l'enjeu sera de générer des liaisons non envisagées jusque-là.

C'est grâce à une analyse du langage que ces liaisons vont être rendues perceptibles. En effet, c'est le discours tenu par une personne qui nous donne ici un accès à sa pensée et, ce faisant, à des recadrages possibles de celle-ci. Ainsi, «la personne qui réalise une mise au carré écoute son interlocuteur et se représente mentalement (ou éventuellement par écrit) ses propos. [...] Elle les saisit mieux et perçoit aussi plus clairement les alternatives " (Faulx et Danse, 2017, p. 26).

\section{Démarche}

La démarche se pratique en trois étapes. Pour la clarté du propos, nous allons les illustrer au départ par ces extraits pris dans différents entretiens avec une même cliente. 
- "Je n'ai pas fait d'études universitaires. Lors des conversations avec mon amie pédiatre, je me sens toujours obligée de prouver quelque chose parce que je me sens inférieure. ”

- "J'ai honte de mon parcours scolaire. Je me sens nulle."

- «J'ai redoublé ma troisième secondaire, hein! » accompagné d'un silence et d'un regard insistants.

Tableau 1. Exemples de liaisons

\section{Étape 1. Repérer une liaison et identifier les deux éléments qui la composent}

Dans l'exemple, l'élément 1 serait "se sentir nulle» et l'élément 2 "avoir eu un parcours scolaire chaotique».

On dispose alors des ingrédients nécessaires pour mener sa démarche: un premier élément, un deuxième élément, une liaison. Dans l'exemple: "être nulle" (élément 1), "avoir eu un parcours scolaire chaotique" (élément 2), une liaison (dont la nature sera précisée plus bas).

Ces éléments liés entre eux peuvent être des objets, des faits, des événements, des phénomènes, des sentiments, des valeurs... Ici, ce sont un jugement sur soi (être nulle) et un phénomène (un parcours scolaire chaotique).

Notons que les liaisons peuvent prendre plusieurs formes, que nous reprenons avec l'exemple:

- cause à effet (l'élément 1 cause l'élément 2/c'est parce que j'ai eu un parcours chaotique à l'école que je me sens nulle);

- cooccurrence (1 et 2 vont ensemble/mon sentiment de nullité et mon parcours chaotique sont apparus ensemble);

- similitude (1 et 2 se ressemblent, ils répondent aux mêmes processus, fonctionnements, lois.../mon sentiment de nullité et le caractère chaotique de ma scolarité se ressemblent, c'est toujours cette difficulté à me faire du bien);

- composante (1 est un élément de 2/mon parcours scolaire chaotique est une des raisons qui fait que je me sens nulle);

- cause indirecte ( 2 vient après $1 /$ mon parcours scolaire chaotique a provoqué pas mal de difficultés dans ma vie, qui ont conduit à ce que je me sente nulle);

- condition (il faut 1 pour avoir 2/pour se sentir nulle, il faut avoir eu un parcours chaotique);

- facteur favorable (1 est un facteur qui favorise $2 /$ se sentir nulle est favorisé par un parcours scolaire chaotique);

- corrélation (plus il y a de 1 , plus il y a de 2/plus le parcours est chaotique, plus on se sent nul). 


\section{Étape 2. Décliner chaque élément en deux critères opposés}

À la manière de ce que font les chercheurs qui mobilisent l'analyse structurale (Piret, Nizet et Bourgeois, 1996). On aboutit alors à quatre critères.

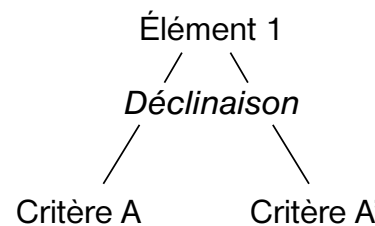

Figure 1. Éléments et critères

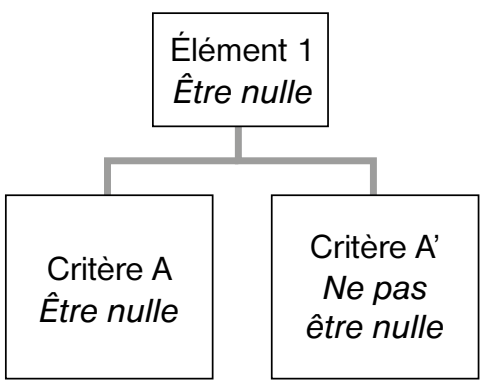

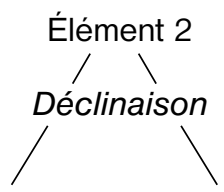

Critère B Critère B'

Figure 2. Éléments et critères exemplifiés

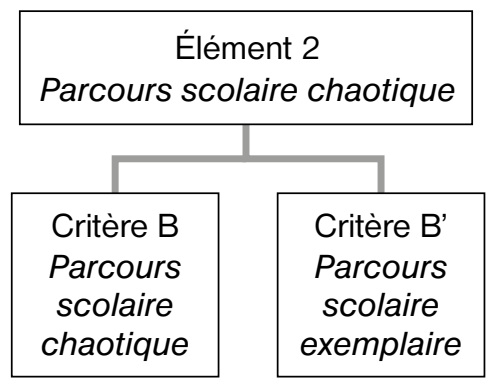

\section{Étape 3. Croiser les quatre possibilités issues}

des quatre critères sous forme d'un tableau

Sachant que la première case représente toujours l'assertion initiale.

On aboutit donc à une visualisation-schématisation de la pensée sous forme d'un tableau à double entrée dont la première case correspond à la représentation exprimée dans le discours initial et dont les trois autres nourrissent l'ambition créative ou heuristique du modèle en offrant des alternatives à cette première case. Ce faisant, cela fait émerger d'autres liaisons possibles mais non envisagées jusqu'alors. Le tableau 2 éclaire les différentes possibilités de liaisons induites par le locuteur.

\begin{tabular}{|l|l|l|}
\hline & Critère B de l'élément 2 & Critère B' de l'élément 2 \\
\hline Critère A de l'élément 1 & $\begin{array}{l}\text { Case 1 } \\
\text { A-B, correspondant à l'association } \\
\text { présente dans le discours initial }\end{array}$ & $\begin{array}{l}\text { Case 2 } \\
\text { A-B', nouvelle possibilité }\end{array}$ \\
\hline Critère A' de l'élément 1 & $\begin{array}{l}\text { Case 3 } \\
\text { A'-B, nouvelle possibilité }\end{array}$ & $\begin{array}{l}\text { Case 4 } \\
\text { A'-B', nouvelle possibilité }\end{array}$ \\
\hline
\end{tabular}

Tableau 2. Mise au carré des critères 
Dans l'exemple, les nouvelles possibilités sont formulées de manière interrogative pour bien signifier leur dimension exploratoire.

\begin{tabular}{|l|l|l|}
\hline & $\begin{array}{l}\text { Critère B de l'élément 2 } \\
\text { Avoir un parcours scolaire } \\
\text { chaotique }\end{array}$ & $\begin{array}{l}\text { Critère B' de l'élément 2 } \\
\text { Avoir un parcours scolaire } \\
\text { adéquat, exemplaire }\end{array}$ \\
\hline $\begin{array}{l}\text { Critère A de l'élément A: } \\
\text { être nul(le) }\end{array}$ & $\begin{array}{l}\text { Case 1 } \\
\text { Assertion initiale : «Je suis nulle, j'ai } \\
\text { doublé " ou «Je suis incompétente, } \\
\text { j'ai un parcours scolaire chaotique » } \\
\text { ou encore «Je suis bête, je n'ai pas } \\
\text { fait d'études universitaires" }\end{array}$ & $\begin{array}{l}\text { Case 2 } \\
\text { être (ou se sentir) nul malgré } \\
\text { un parcours exemplaire? }\end{array}$ \\
\hline $\begin{array}{l}\text { Critère A' de l'élément A: } \\
\text { ne pas être nul(le) }\end{array}$ & $\begin{array}{l}\text { Case 3 } \\
\text { Nouvelle possibilité: peut-on ne } \\
\text { pas être (ou ne pas se sentir) nul } \\
\text { tout en ayant eu un parcours sco- } \\
\text { laire chaotique? }\end{array}$ & $\begin{array}{l}\text { Nouvelle possibilité : peut-on } \\
\text { vivre un parcours exemplaire } \\
\text { et ne pas être nul? }\end{array}$ \\
\hline
\end{tabular}

Tableau 3. Mise au carré des critères de l'exemple introductif

Au-delà du discours initial apparaissent donc trois nouvelles possibilités, trois nouvelles manières de voir une situation ouvrant ainsi sur des versants créatifs et heuristiques propices aux recadrages.

À l'intérieur de chacune des cases, on peut investiguer deux registres de précisions: les situations et les conditions.

Les situations, ce sont les cas de figure, les exemples, les moments, les événements, les personnes pour lesquelles la configuration identifiée s'est présentée. Par exemple, il s'agirait d'inventorier en case 3 différentes personnes (connues de Lila) qui ne se sentent pas nulles et qui pourtant ont eu un parcours scolaire chaotique. Ou de se pencher sur les fois où, malgré son parcours chaotique, elle ne s'est pas sentie nulle.

Les conditions correspondent aux caractéristiques des situations comprises dans une même case. Ce sont des circonstances qui ont rendu cette configuration possible. Dans l'exemple, en case 3, il s'agirait d'investiguer ce qui a permis, pour ces différentes personnes (ou pour elle), que soit associé le fait de ne pas se sentir nul et d'avoir eu un parcours scolaire chaotique. Cela pourrait être de vivre aujourd'hui des situations gratifiantes, de bénéficier de renforcements positifs de la part de l'environnement, d'être heureux dans d'autres domaines de la vie, de vivre dans un milieu qui n'accorde que peu d'importance à la scolarité, etc. 


\section{Une approche typiquement systémique \\ des problèmes humains}

En quoi la mise au carré s'intègre-t-elle typiquement dans l'esprit de la thérapie systémique? Tout d'abord, on trouve comme fondement de l'approche thérapeutique systémique que, souvent, c'est la définition même d'un problème qui rend celui-ci insoluble (Haley, 1997; Faulx, 2006). Travailler les assertions ou les croyances pour amener la personne ou la famille à en concevoir d'autres définitions est donc essentiel dans cette optique. Or, celles-ci sont fortement marquées par un ensemble d'associations que la mise au carré va s'employer à démonter.

Ensuite, la mise en lumière de patterns interactionnels (Wajeman, 2005) constitue un autre processus essentiel de la démarche thérapeutique systémique. Ces patterns interactionnels se donnent notamment à voir à travers le discours, à travers les associations systématiques qui sont portées par les personnes et s'incarnent dans leur narration. Les repérer et les faire évoluer, c'est donc aussi se donner l'occasion de questionner le pattern.

Enfin, il faut rappeler avec Malarewicz (2000) qu'il n'est pas de changement sans changement de point de vue. Ce que propose la mise au carré, et de manière générale toute démarche de recadrage, c'est de trouver des portes d'entrée vers une déstabilisation des représentations, une autre manière de dire les choses et de les penser.

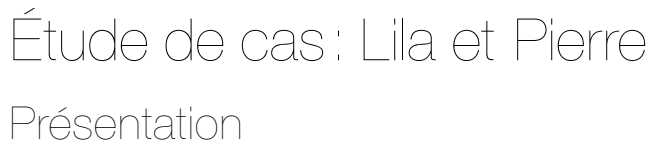

Lila, 47 ans, se présente avec son mari, Pierre, 49 ans. Ils sont parents de deux grandes adolescentes. Lila arrive en consultation fatiguée et stressée. Elle prend des antidépresseurs et revient d'un congé maladie de trois semaines. Quatre mois auparavant, elle a vécu le décès de son père, mort des suites d'un cancer. Elle est hantée par des souvenirs douloureux: tous les jours lui reviennent les images de son papa étendu sur son lit de mort. Elle connaît également des difficultés d'organisation de sa vie professionnelle liées à la disparition du soutien que lui apportait son père. Pierre a accepté de se rendre à la consultation avec son épouse car il dit "ressentir sa grande souffrance". Cela crée aussi selon lui des tensions tant au restaurant qu'ils gèrent ensemble qu'en famille.

Au cours du premier entretien, Lila décrit sa tristesse. Elle fait part de ses difficultés à s'endormir: les nuits, elle voit son père dans ses rêves, se réveille et ne parvient plus à retrouver le sommeil. Régulièrement, des maux de tête surgissent. Chaque soir, pour trouver de l'apaisement, elle boit un ou deux verres, habitude qui s'est progressivement installée depuis le décès. Pierre le déplore mais l'accepte. 
Il y a un an, Pierre a ouvert son propre restaurant. Lila explique combien ça a été éprouvant - et ça l'est toujours au moment de la consultation - de jongler entre son propre emploi (employée administrative) et les week-ends à travailler au restaurant.

Dans le système professionnel (le restaurant) comme dans le système familial, Lila se sent en difficulté. Elle dit être sous pression et courir après le temps et ne plus trouver sa place. Les incidents avec Pierre se multiplient.

Lila se dit "à bout" et a l'impression qu'elle "ne parvient/parviendra pas à tout gérer". Son but est de faire le deuil de son papa comme annoncé au début du premier entretien et aussi de se re-sentir bien au niveau de ses relations tant professionnelles que privées.

\section{Une première mise au carré}

Dans les propos de Lila, on relève l'expression souvent employée: "Je n'y arriverai pas", associée à la gestion du restaurant (ainsi qu'à la gestion du quotidien et à sa vie au bureau). La mise au carré va nous permettre de construire des recadrages afin d'accompagner Lila.

Voici comment a été construite la première mise au carré. Nous sommes d'abord partis de plusieurs assertions initiales sur sa place dans le système qui indiquent une liaison: d'un côté, "je ne me fais pas confiance" (formulé aussi "je ne me sens pas capable», "je n’y arriverai pas"...), associé avec «je ne gère pas correctement le restaurant" (formulé aussi "la vaisselle n'a pas été faite au fur et à mesure de la soirée", "on a pris trop de réservations pour une même soirée", "je travaille mal pour le moment").

Mis ensemble, ces deux éléments ont donné des affirmations du type: "en me faisant si peu confiance, je ne peux pas arriver à gérer correctement le restaurant" ou, chez Pierre, "évidemment, si tu te fais si peu confiance, cela ne risque pas de s'améliorer".

On tient donc là ce qui pourrait être l'assertion initiale. Elle est composée de deux éléments de départ:

- A: ne pas gérer;

- B: ne pas se faire confiance.

Si on décline chacun des éléments qui la composent, cela donne:

- A: ne pas gérer/A': gérer;

- B: ne pas se faire confiance/B': se faire confiance.

Trois zones à explorer, porteuses potentiellement de recadrages, s'ouvrent donc grâce à la mise au carré: 


\begin{tabular}{|c|c|c|}
\hline & $\begin{array}{l}\text { B: Ne pas se faire confiance } \\
\text { «Je n'y arriverai pas" }\end{array}$ & $\begin{array}{l}\text { B': Se faire confiance } \\
\text { «Je peux y arriver» }\end{array}$ \\
\hline A: Ne pas gérer & $\begin{array}{l}\text { Case } 1 \\
\text { Position initiale: «Je ne suis pas capable } \\
\text { de gérer les week-ends au restaurant car } \\
\text { je ne me fais pas confiance» } \\
\text { "Si tu n'y crois pas, tu n'y arriveras jamais » } \\
\text { "À force de ne pas y arriver, ça n'aide pas } \\
\text { à se sentir forte» }\end{array}$ & $\begin{array}{l}\text { Case } 2 \\
\text { Recadrage } 1 \text { : On peut mal gérer } \\
\text { des situations et pourtant se } \\
\text { faire confiance. Ou (variante } \\
\text { possible): bien qu'on se fasse } \\
\text { confiance, on peut mal gérer. }\end{array}$ \\
\hline$A^{\prime}$ : Gérer & $\begin{array}{l}\text { Case } 3 \\
\text { Recadrage } 2 \text { : On peut bien gérer des } \\
\text { situations et pourtant ne pas se faire } \\
\text { confiance. Ou (variante possible) : bien } \\
\text { qu'on ne se fasse pas confiance, on peut } \\
\text { y arriver. }\end{array}$ & $\begin{array}{l}\text { Case } 4 \\
\text { Recadrage } 3 \text { : On peut gérer des } \\
\text { situations et se faire confiance. } \\
\text { Ou (variante possible): Je peux } \\
\text { gérer des situations et me faire } \\
\text { confiance. }\end{array}$ \\
\hline
\end{tabular}

Tableau 4. Mise au carré "gérer" - "ne pas se faire confiance"

Nous allons maintenant décrire les différents recadrages auxquels nous avons procédé.

Case 2 (recadrage 1)

\begin{tabular}{|l|l|l|}
\hline & $\begin{array}{l}\text { B: Ne pas se faire confiance } \\
\text { «Je n'y arriverai pas» }\end{array}$ & $\begin{array}{l}\text { B': Se faire confiance } \\
\text { «Je peux y arriver » }\end{array}$ \\
\hline A: Ne pas gérer & $\begin{array}{l}\text { Case 1 } \\
\text { Position initiale }\end{array}$ & $\begin{array}{l}\text { Case 2 } \\
\text { Recadrage 1: On peut mal gérer des } \\
\text { situations et pourtant se faire confiance. }\end{array}$ \\
\hline A': Gérer & $\begin{array}{l}\text { Case 3 } \\
\text { Recadrage 2 }\end{array}$ & $\begin{array}{l}\text { Case 4 } \\
\text { Recadrage horizon 3 }\end{array}$ \\
\hline
\end{tabular}

Tableau 5. Mise au carré "gérer» - «ne pas se faire confiance», recadrage 1

Le premier recadrage visé se situe en case 2 . Cette case va nous aider à envisager un cas de figure qui se situe actuellement hors du cadre de pensée du couple. En effet, Pierre et Lila ne parviennent pas (ou plus) à se connecter à la possibilité suivante: malgré une confiance en soi, on peut se trouver débordé par certaines situations. L'enjeu est de dépasser une forme de pensée magique: quand on a confiance en soi, on vient à bout de tout! Pour aider à voir différemment, on va explorer avec elle des situations où la confiance ne suffit pas, ne prémunissant pas de l'échec ou de la difficulté.

On peut y arriver avec des questions du style:

- Vous diriez en général que les gens qui se font confiance surmontent toutes les situations?

- Est-ce que, pour vous, avoir confiance en soi, c'est comme une garantie de succès?

- Est-ce que vous avez connu des situations où, bien que vous ayez confiance en vous, cela n'a pas donné les résultats escomptés? 
Cela nous a permis de travailler les situations et les conditions de cette case 2:

- Les situations. Nous avons invité Lila et Pierre à évoquer des cas dans lesquels elle ou d'autres personnes ne sont pas parvenues à gérer des situations en dépit d'une bonne confiance en elles-mêmes. Dans le cas présent, on a notamment procédé par décentration en aidant à visualiser des personnes confiantes en elles qui pourtant ont échoué dans leurs entreprises. Cela contribuera à déconstruire le lien entre les deux éléments et donc à ouvrir l'espace des possibles. Et de fait, Lila évoque notamment à son frère que rien n'effraie et dont les entreprises n'ont pourtant pas été couronnées de succès.

- Les conditions. Une fois fait, on peut prolonger le recadrage en investiguant les conditions qui ont stimulé ce type de configuration. Autrement dit, qu'est-ce qui fait que des personnes qui ont confiance en elles échouent dans leurs entreprises? Parmi les éléments identifiés par Lila et Pierre, on peut citer: une tâche très complexe, un manque de préparation avant de se lancer, une situation inédite à laquelle on n'a jamais été confronté, un travail collectif dans lequel les rôles et fonctions sont peu définis, une incompétence professionnelle et non personnelle...

Ce travail nous a amenés à un deuxième recadrage fondé sur la case 3 .

Case 3 (recadrage 2)

\begin{tabular}{|c|c|c|}
\hline & $\begin{array}{l}\text { B: Ne pas se faire confiance } \\
\text { «Je n'y arriverai pas" }\end{array}$ & $\begin{array}{l}\text { B': Se faire confiance } \\
\text { «Je peux y arriver» }\end{array}$ \\
\hline A: Ne pas gérer & $\begin{array}{l}\text { Case } 1 \\
\text { Position initiale }\end{array}$ & $\begin{array}{l}\text { Case } 2 \\
\text { Recadrage } 1\end{array}$ \\
\hline$A^{\prime}$ : Gérer & $\begin{array}{l}\text { Case } 3 \\
\text { Recadrage } 2 \text { : On peut bien gérer des situations } \\
\text { et pourtant ne pas se faire confiance. }\end{array}$ & $\begin{array}{l}\text { Case } 4 \\
\text { Recadrage } 3\end{array}$ \\
\hline
\end{tabular}

Tableau 6. Mise au carré "gérer» - "ne pas se faire confiance», recadrage 2

Schématiquement, ce recadrage peut s'exprimer ainsi : on peut gérer des situations difficiles et pourtant ne pas se faire confiance. Le recadrage commence par étudier avec elle les situations et les conditions dans lesquelles elle est parvenue à gérer correctement une situation malgré une confiance défaillante.

Les situations. Lila va se remémorer avec Pierre des moments, voire des services pour lesquels elle s'en est "plutôt bien tirée, tout compte fait ". Pierre: "Il y a eu des week-ends où malgré la peur tout s'est bien passé. " Lila: "J'y arrive de mieux en mieux malgré tout."

À cette occasion, notons que les recadrages vis-à-vis du restaurant sont similaires pour le système professionnel. C'est comme si, pour Lila, les recadrages liés au restaurant étaient transférés progressivement aux autres contextes de sa vie. Ainsi, malgré le manque de confiance qui tend à persister, elle constate qu'elle gère de mieux en mieux non seulement les «coups de feu " et les moments 
de stress au travail, mais aussi les tensions familiales, les difficultés privées. Si pour le système professionnel, elle dira "ça revient", elle rapportera également que sa fille a remarqué des changements. Sa maman lui semble "plus posée". Sont-ce là les prémisses de la case 4 ? Nous y reviendrons plus tard.

Les conditions. Lorsque nous réfléchissons avec le couple aux conditions qui ont rendu possibles ces situations au restaurant, nous relevons avec eux des éléments de nature aussi bien psychologique (accepter de déléguer, voir et apprécier l'aide apportée au restaurant par les autres membres de la famille) qu'organisationnelle (l'engagement de personnel en fonction des besoins et des possibilités financières, l'attribution de rôles à chacun, établissement d'un équilibre raisonnable entre acceptation des réservations et possibilités d'accueil...). Du côté du contexte familial, nous identifions également des conditions facilitatrices: accepter de voir ses enfants grandir et dès lors leur donner la possibilité de prendre plus de place dans les tâches familiales, mettre des limites, prendre du temps pour soi... Et par la même occasion, retrouver sa place au travail et revivre des moments en famille agréables.

On l'aura compris, il importe donc ici de se pencher sur la manière de gérer sans pour autant viser à tout prix cette fameuse confiance à laquelle Lila prête tant de pouvoir ou en tout cas qu'elle semble associer à l'efficacité professionnelle et familiale.

Case 4 (recadrage 3)

\begin{tabular}{|l|l|l|}
\hline & $\begin{array}{l}\text { B: Ne pas se faire confiance } \\
\text { «Je n'y arriverai pas » }\end{array}$ & $\begin{array}{l}\text { B': Se faire confiance } \\
\text { «Je peux y arriver» }\end{array}$ \\
\hline A: Ne pas gérer & $\begin{array}{l}\text { Case 1 } \\
\text { Position initiale }\end{array}$ & $\begin{array}{l}\text { Case 2 } \\
\text { Recadrage 1 }\end{array}$ \\
\hline A': Gérer & $\begin{array}{l}\text { Case 3 } \\
\text { Recadrage 2 }\end{array}$ & $\begin{array}{l}\text { Case 4 } \\
\text { Recadrage 3: On peut gérer des } \\
\text { situations et se faire confiance. }\end{array}$ \\
\hline
\end{tabular}

Tableau 7. Mise au carré "gérer" - «ne pas se faire confiance», recadrage 3

Plus tard, dans la thérapie, on pourra arriver au troisième recadrage: Lila peut "gérer" et "se faire confiance". Ce dernier recadrage, du point de vue de sa vision du monde, est le plus proche de l'assertion initiale puisque si l'on considère que "ne pas gérer" va avec "ne pas avoir confiance", il est très probable que, dans cette vision du monde, "gérer" aille avec "avoir confiance".

En revanche, il s'agit d'un recadrage plus profond car à l'opposé des croyances familiales. Là, l'enjeu sera donc d'ouvrir sur la possibilité de se situer dans la case "avoir confiance" et "gérer correctement". Cela constitue une perspective plus lointaine, que nous pourrions définir comme un recadrage "horizon" au sens des "concepts horizons" qui définissent une "sorte d'idéal qui sert de référence pour penser la réalité» (Paugam, 1996). Cela définit une perspective mais pas un but car, comme chacun sait, on n'atteint jamais l'horizon. Vu l'ampleur du trajet à parcourir, une telle modification de sa vision d'elle-même 
et du monde aurait été impossible sans mener le chemin décrit. Par contre, en faisant le détour par les cases 2 et 3, Lila a pu envisager des possibilités de (se) penser autrement. "Maintenant, je me sens capable, même dans les moments chauds."

Notons au passage que nous avons relaté ici une mise au carré qui suivait un ordre particulier. Nous sommes partis de l'assertion initiale en case 1 pour proposer ensuite trois recadrages respectivement dans les cases 2,3 et puis 4 .

\begin{tabular}{|l|l|l|}
\hline & $\begin{array}{l}\text { B: Ne pas se faire confiance } \\
\text { «Je n'y arriverai pas" }\end{array}$ & $\begin{array}{l}\text { B': Se faire confiance } \\
\text { «Je peux y arriver " }\end{array}$ \\
\hline A: Ne pas gérer & $\begin{array}{l}\text { Case 1 } \\
\text { Position initiale }\end{array}$ & $\begin{array}{l}\text { Case 2 } \\
\text { Recadrage 1 }\end{array}$ \\
\hline A': Gérer & $\begin{array}{l}\text { Case 3 } \\
\text { Recadrage 2 }\end{array}$ & $\begin{array}{l}\text { Case 4 } \\
\text { Recadrage horizon 3 }\end{array}$ \\
\hline
\end{tabular}

Tableau 8. Mise au carré "gérer» - «ne pas se faire confiance», recadrage " horizon"

La progression aurait pu s'imaginer dans un autre ordre. Nous aurions pu commencer le recadrage en case 3 pour aider Lila à prendre conscience des situations qu'elle parvenait à gérer malgré son manque de confiance. Nous aurions pu ensuite aller vers la case 2 afin de travailler sur des situations où on ne gère pas bien malgré une confiance en elle. Nous aurions terminé également en case 4 .

Voici ce que ce trajet aurait donné:

- Je ne gère pas car je ne me fais pas confiance (case 1).

- Pourtant, il est possible de gérer tout en n'ayant pas confiance (recadrage 2, case 3).

- Par ailleurs, des personnes qui se font confiance ne gèrent pas nécessairement (recadrage 1, case 2).

- Je pourrais donc me faire confiance et y arriver (recadrage horizon 3, case 4).

\begin{tabular}{|l|l|l|}
\hline & $\begin{array}{l}\text { B: Ne pas se faire confiance } \\
\text { «Je n'y arriverai pas» }\end{array}$ & $\begin{array}{l}\text { B': Se faire confiance } \\
\text { "Je peux y arriver » }\end{array}$ \\
\hline A: Ne pas gérer & $\begin{array}{l}\text { Case 1 } \\
\text { Position initiale }\end{array}$ & $\begin{array}{l}\text { Case 2 } \\
\text { Recadrage 1 }\end{array}$ \\
\hline A': Gérer & $\begin{array}{l}\text { Case 3 } \\
\text { Recadrage 2 }\end{array}$ & $\begin{array}{l}\text { Case 4 } \\
\text { Recadrage horizon 3 }\end{array}$ \\
\hline
\end{tabular}

Tableau 9. "Gérer» - «ne pas se faire confiance», exemple de trajet 1 
On aurait pu, de manière plus directe, ne pas passer par la case 2 et aller directement vers la case 3 et la case 4 , en abordant directement les situations de réussite.

Voici ce que ce trajet aurait donné:

- Je ne gère pas car je ne me fais pas confiance (case 1).

- Pourtant, il est possible de gérer tout en n'ayant pas confiance (recadrage 2, case 3).

- Je pourrais donc me faire confiance et y arriver (recadrage horizon 3, case 4).

\begin{tabular}{|l|l|l|}
\hline & $\begin{array}{l}\text { B: Ne pas se faire confiance } \\
\text { «Je n'y arriverai pas» }\end{array}$ & $\begin{array}{l}\text { B': Se faire confiance } \\
\text { «Je peux y arriver }\end{array}$ \\
\hline A: Ne pas gérer & $\begin{array}{l}\text { Case 1 } \\
\text { Position initiale }\end{array}$ & $\begin{array}{l}\text { Case 2 } \\
\text { Recadrage 1 }\end{array}$ \\
\hline A': Gérer & $\begin{array}{l}\text { Case 3 } \\
\text { Recadrage 2 }\end{array}$ & $\begin{array}{l}\text { Case 4 } \\
\text { Recadrage horizon 3 }\end{array}$ \\
\hline
\end{tabular}

Tableau 10. "Gérer» - "ne pas se faire confiance», exemple de trajet 2

Le choix de ce détour dépend aussi de ce que le client amène, de sa réceptivité, de sa vision du monde. Nous en reparlons dans la discussion.

\section{Une deuxième mise au carré}

Suite aux différents recadrages, Lila estime désormais qu'elle peut se faire confiance pour agir dans des situations difficiles que ce soit dans le domaine professionnel ou familial. Elle ne fait plus d'insomnies et ne rumine plus la nuit. Elle est moins fatiguée. En outre, ses maux de tête se sont estompés.

Le premier recadrage a ouvert les possibilités d'un deuxième recadrage par une autre mise au carré. En effet, après ce travail fait avec elle, elle réévoque un élément qu'elle avait abordé au début de la thérapie: le deuil de son papa. Si elle estime déjà avoir fait du chemin à ce sujet ("je suis dans un autre état d'esprit»), elle sent néanmoins que ce point entrave son bien-être. C'est comme si le premier travail facilité par la mise au carré avait permis de traiter un premier "volet" et laissait la place à un autre travail, celui-là centré sur la question du deuil de son père.

C'est également en nous aidant de la mise au carré que nous avons abordé la suite de la thérapie. En effet, nous repérons une association entre la mort de son papa et une difficulté (voire une impossibilité) de faire face au quotidien.

En lui posant la question de savoir ce qui est difficile pour elle par rapport à ce deuil, elle nous explique: "Sans lui, je perds mes repères"; "sans lui, je suis incapable d'agir, vivre, penser, être". Sans ce "modèle", elle a l'impression qu'elle ne peut pas "affronter le monde», elle est "bloquée». 


\begin{tabular}{|l|l|l|}
\hline & $\begin{array}{l}\text { B: Ne pas faire face au monde } \\
\text { Ne pas gérer }\end{array}$ & $\begin{array}{l}\text { B': Faire face au monde } \\
\text { Gérer }\end{array}$ \\
\hline $\begin{array}{l}\text { A: Papa mourant/ } \\
\text { décédé }\end{array}$ & $\begin{array}{l}\text { Case 1 } \\
\text { Position initiale: «Sans mon papa, je ne } \\
\text { peux pas faire face, je ne peux pas gérer » } \\
\text { «J'ai perdu ma boussole, je ne sais plus } \\
\text { où je vais" }\end{array}$ & $\begin{array}{l}\text { Case 2 } \\
\text { Recadrage 2: Malgré } \\
\text { l'absence/le décès de } \\
\text { mon papa, je peux gérer }\end{array}$ \\
\hline A': Papa vivant & $\begin{array}{l}\text { Case 3 } \\
\text { Recadrage 1: Même si mon papa est } \\
\text { vivant, je peux avoir des difficultés à gérer }\end{array}$ & $\begin{array}{l}\text { On fait face à la vie en ayant } \\
\text { son papa à ses côtés }\end{array}$ \\
\hline
\end{tabular}

Tableau 11. Mise au carré "papa décédé» - "ne pas faire face au monde»

Dans l'usage thérapeutique de cette mise au carré, un ordre de recadrage nous a semblé plus favorable que les autres:

- de la position initiale: mon papa décédé, je ne peux plus gérer ma vie (position initiale, case 1);

- au recadrage 1 en case 3 : mon papa vivant, je ne gérais pas nécessairement tout si facilement;

- pour ensuite passer au recadrage 2 en case 2 : malgré le décès de mon papa, je peux gérer (recadrage 1, case 2 ).

La case 3 permet un détour avant d'arriver à la case 2 qui est en quelque sorte l'objectif de Lila, sa perspective.

\begin{tabular}{|l|l|l|}
\hline & $\begin{array}{l}\text { B: Ne pas faire face } \\
\text { Ne pas gérer }\end{array}$ & $\begin{array}{l}\text { B': Faire face } \\
\text { Gérer }\end{array}$ \\
\hline $\begin{array}{l}\text { A: Papa mourant/ } \\
\text { décédé }\end{array}$ & $\begin{array}{l}\text { Case 1 } \\
\text { Position initiale }\end{array}$ & $\begin{array}{l}\text { Case 2 } \\
\text { Recadrage 1 }\end{array}$ \\
\hline A': Papa vivant & $\begin{array}{l}\text { Case 3 } \\
\text { Recadrage 2 }\end{array}$ & $\begin{array}{l}\text { Case 4 } \\
\text { Recadrage 3 }\end{array}$ \\
\hline
\end{tabular}

Tableau 12. Mise au carré "papa décédé» - "ne pas faire face au monde», recadrages

Décrivons le processus un peu plus précisément.

Au départ de la case 1, Lila associe le décès de son papa à l'impossibilité de faire face à la vie, elle l'exprime comme ceci: "Mon papa est comme une béquille, sans lui je ne peux plus avancer [...] je ne sais plus affronter la vie.»

Par un détour via la case 3 , Lila est amenée à prendre un premier recul par rapport à son assertion initiale. L'idée consiste à trouver des exemples où elle ou d'autres se sont retrouvés dans l'impossibilité de faire face à la vie malgré un papa vivant. Elle va pouvoir se remémorer des situations où elle a éprouvé des difficultés à gérer sa vie alors même que son père était présent. Reviennent alors à la mémoire: des moments d'adolescence difficiles, des relations amicales ou amoureuses conflictuelles... Du coup, bien que son papa ait 
été présent dans ces moments-là de sa vie, Lila a été seule pour y faire face. Son papa n'a pas assumé ces épreuves à sa place, elle a dû les gérer elle-même. Son assertion initiale peut déjà se délier un peu grâce à ce recadrage.

Quelles conditions peuvent générer ces situations? Elle liste des éléments comme le manque de "compétences relationnelles" des pères, le fait qu'ils n'ont pas toujours l'envie ou le temps d'aider, ou encore un passage obligé de la vie au sens du rite ou de la transition.

Le constat peut sembler une évidence mais cela ne l'est pas tant pour elle: que son papa soit vivant (ou non), Lila n'est pas à l'abri d'une gestion difficile et éprouvante du quotidien. Avec ce recadrage, elle chemine vers cette nouvelle idée.

Ce passage par la case 3 prépare le recadrage de la case 2 : gérer sa vie malgré le décès de son papa. Étant à l'opposé de son assertion initiale, il n'aurait probablement pas pu être travaillé directement. Dans son discours, on peut noter des indices de son évolution: "Je ne ressens plus de profonde tristesse. Par contre, j'ai toujours mal au cœur même si je n'ai plus d'angoisses ", "Je me sens bien mieux". Ces indices langagiers nous font dire que Lila a maintenant les ressources pour poursuivre la mise au carré. Ceci alimente notre hypothèse qu'il aurait été trop brutal, suite à son assertion initiale, de lui proposer de travailler sur le lien "papa décédé" et "possibilité de gérer".

Ce recadrage de la case 2 associe le fait de pouvoir faire face aux événements de la vie alors même que son papa est décédé ou du moins pas là. Elle a déjà pu le prouver à plusieurs reprises dans plusieurs situations. Par exemple, quand son papa se déplaçait à l'étranger, quand elle a quitté le domicile familial pour créer sa propre famille, et depuis quelque temps elle fait face à la vie alors que son papa est parti.

Son papa "reste" certes un pilier dans sa vie, mais il n'est pas la seule ressource disponible pour Lila. Maintenant, elle s'en aperçoit et peut dorénavant en activer d'autres:

- au restaurant et à la maison, elle a pu déléguer des responsabilités à son mari et à ses enfants. Au niveau familial, elle peut compter sur sa maman et sa fratrie. Elles se soutiennent l'une l'autre;

- au bureau, dorénavant, "les jeunes collègues font leur part" même s'ils restent hyperconnectés;

- aussi, Lila peut se voir elle-même comme une ressource. Elle reprend sa place de femme adulte qui sait gérer et faire face là où elle restait ancrée dans le rôle de "la petite fille modèle de son papa".

Les mises au carré ont ouvert la voie à beaucoup de changements. Dernièrement, elle se dit "beaucoup mieux, plus calme, plus posée, plus souriante...". Avec le suivi de son médecin, elle arrête progressivement les antidépresseurs.

Comme si cela coulait de source, au final, elle a pu se sentir capable de "voler de ses propres ailes" en confiance, pour reprendre sa propre expression lors de la dernière séance. 
Dans ce cas-ci, le recadrage 3 de la case 4 «Si mon papa était en vie, je pourrais bien gérer ma vie" vient confirmer son assertion initiale. Dans ce cadre thérapeutique, il n'a pas été travaillé, alimentant une nostalgie, ce qui n'empêche qu'il puisse être intéressant aussi à traiter comme tel.

\section{Discussion}

\section{Les deux bénéfices de la mise au carré}

Les bénéfices de la mise au carré ont déjà été discutés pour la recherche qualitative dans l'article de Fossion et al. (2018). On y apprend que, lorsqu'elle est utilisée dans ce contexte, cette technique présente deux intérêts principaux: (1) une meilleure compréhension des propos de la personne qui s'exprime et (2) l'émergence de nouvelles hypothèses. Transférés ici à la pratique clinique et d'accompagnement, ces deux aspects se retrouvent, bien que de manière un peu différente. Dans la pratique clinique, le premier pan, celui de la compréhension, s'incarne dans le fait que cette démarche va aider tout d'abord à vérifier les frontières de ce qui est dit, afin de saisir, avec précision, les contours de la pensée de la personne rencontrée. De l'autre côté, plutôt que d'émergence de nouvelles hypothèses, on parlera ici d'exploration de nouvelles idées ou nouveaux moyens d'action.

Ainsi, le passage par la mise au carré permet tout d'abord de mieux comprendre et situer les propos de l'interlocuteur. Lorsque Lila nous dit qu'elle ne parvient pas à gérer les situations depuis que son papa n'est plus là, avant même d'envisager un recadrage, le fait de lui demander, par exemple, s'il existe des situations qui font exception à cette règle nous fait percevoir l'étendue des propos. On comprend mieux quels cas sont visés quand elle dit ne pas pouvoir faire face. On identifie par exemple qu'il s'agit surtout de situations à caractère public (travailler, gérer un restaurant, réussir des examens) alors que des situations à caractère privé (accepter la séparation avec un petit ami, gérer un conflit amical) sont moins concernées. Comme en recherche, cela donne l'occasion de cheminer par le questionnement pour affiner la compréhension du sens (Fossion et al., 2018).

Le deuxième aspect, celui de l'émergence de nouvelles hypothèses, relève ici de l'apport de pistes de réflexions ou d'actions nouvelles au service de la personne rencontrée. Lila envisage par exemple de nouvelles manières de considérer la place de son père dans sa vie et de nouvelles façons de faire au restaurant.

De la sorte, la mise au carré fournit à l'intervenant une méthodologie pour produire de recadrages, ceux-ci pouvant toucher autant l'action que les représentations qui les sous-tendent. On retrouve ici la dialectique du travail thérapeutique, bien décrite par les rapports entre eusémie et euhérésis par Isebaert et Cabié (2015). Le travail sur les perceptions, les significations, le sens donné aux événements (eusémie) est en effet indissociable du travail sur l'action et les choix posés par la personne (euhérésis). Pour exprimer ce cycle, on peut dire que, d'une part, l'ouverture à d'autres cartes du monde et d'autres perceptions du réel permet à la personne de retrouver une certaine 
liberté d'agir et, de l'autre, en agissant différemment, cela va générer d'autres événements, d'autres réalités, et donc des possibilités de construire de nouvelles représentations.

\section{Partir de ce qui est amené et aller vers les cas atypiques et les exceptions}

Fidèles aux fondements du recadrage et de la pratique systémique, c'est toujours au départ de ce que la personne amène (du monde du client), matérialisé ici en case 1, que les nouvelles pistes pour penser et agir sont travaillées. Dans l'étude de cas, c'est à partir de la narration de Lila et des différents récits qui illustrent l'importance de son père dans sa vie que l'on dessine d'autres manières de penser qui viennent moduler ou relativiser celle-ci.

Cela nous donne à penser que les approches de thérapie narrative peuvent trouver dans la mise au carré une manière utile de créer de nouveaux récits qui viennent offrir des "alternatives aux histoires dominantes" (Mori et Rouan, 2011). De la sorte, on favorise de plus grandes possibilités dans la vie de la personne en lui offrant un répertoire plus large de narrations possibles. On a vu dans le cas présenté ici que cela se fait notamment par un travail sur les situations qui échappent à la routine des représentations et qui dès lors présentent pour Lila un caractère inédit ou rare. Certaines offrent des alternatives à des croyances générales : "avoir confiance» et pourtant "ne pas gérer" ou "ne plus avoir son père" mais "s'en sortir quand même». D'autres concernent davantage des croyances personnelles, avec "ne pas avoir mon père" et " $m$ 'en sortir".

L'idée est donc d'inviter les personnes à raconter des expériences qui ne sont pas "en phase" avec leur histoire dominante, de telle sorte qu'elles réfléchissent à des "événements uniques" ou des "exceptions" (Mori et Rouan, 2011 ; Morgan, 2019).

Si l'exception est au cœur de l'approche narrative (Morgan, 2019), on a vu dans l'introduction qu'elle constitue un point d'ancrage très important également dans les thérapies orientées solution. Ainsi, de Shazer affirme-t-il, reprenant les propos d'Erickson, qu'«il y a toujours une exception" (Betbèze, 2002). Un tel postulat est au cour aussi de la mise au carré. On le voit bien avec Lila, il y a toujours au moins une situation (au sens où cela a été défini dans l'article) dans chacune des cases. Or, cette prise de conscience que les événements ne fonctionnent pas toujours de la même manière est essentielle pour l'activation de solutions. Grâce à cela, on crée un contexte qui augmente les choix, un enjeu central dans le Modèle de Bruges, héritier de l'approche de de Shazer (Isebaert et Cabié, 2015). C'est ce qu'on voit dans l'étude de cas: des possibilités d'agir différentes apparaissent à Lila par exemple dans sa manière de gérer le restaurant sans son père, en activant d'autres ressources. La mise au carré, qui montre que rien n'est jamais binaire car il y a toujours ces cas particuliers qui viennent mettre en doute les raisonnements automatiques, offre donc un moyen pour concrétiser cette intention thérapeutique. Eusémie et euhérésis ont ainsi été mis en mouvement. 


\section{La question des trajets}

Un aspect important de la démarche consiste en l'organisation de trajets de pensée au travers du carré. C'est comme un voyage intellectuel qui s'opère par le jeu des questions avec une mécanique qui fait se combiner les différents critères. La matrice au carré comprend une case de départ, puis des cases visées qui s'atteignent via des cases intermédiaires. Le processus est progressif. Il est difficile de théoriser quels sont les trajets les plus indiqués mais les observations des différents cas décrits peuvent nous aider à tracer quelques généralités. Elles ne remplacent pas la sensibilité et l'intelligence de l'intervenant, bien entendu: il doit pouvoir éprouver à travers l'entretien quelles sont les directions à prendre et à quoi la personne est prête à un moment du processus de changement.

Les indices qui peuvent aider à penser les trajets peuvent s'exprimer en distance symbolique. Ainsi, pour Lila, dans le premier recadrage présenté dans cet article, gérer des situations et se faire confiance semble "loin" de sa case initiale. C'est un recadrage "horizon" parce qu'à l'opposé de sa perception d'elle-même à ce moment du processus. On décide donc dans ce cas d'opter pour une autre combinaison: bien que ne se faisant pas confiance, on peut gérer. Mais à nouveau, cela semble trop loin, nous décidions de passer d'abord par une réflexion plus décentrée sur les personnes qui se font confiance et pourtant ne parviennent pas nécessairement à tout gérer. Cette décentration a permis une évolution beaucoup plus progressive.

\section{Des enchaînements de mises au carré}

On comprend bien dans le cas de Lila que les mises au carré s'enchaînent. Une première donne lieu à une autre, plus personnelle. Nous devons d'ailleurs signaler que d'autres mises au carré ont suivi celles que nous avons présentées, notamment une assez surprenante entre papa vivant (A) - papa mort (A') et papa présent (B) - papa absent (B') qui a ouvert sur des réflexions presque métaphysiques ou spirituelles. Lila en est arrivée à se questionner non seulement sur la présence de son papa lorsqu'il était vivant, mais aussi sur une présence potentielle alors même qu'il est mort, une présence en pensée, en conseil, en souvenirs. La cascade des mises au carré peut donc s'avérer infinie.

\section{Questions d'utilisation}

La mise au carré constitue un appui à l'entretien. Pour autant, on comprend aisément que cette technique ne doit pas être appliquée systématiquement tout au long de celui-ci. En effet, bien qu'elle parte d'une approche compréhensive, elle comporte aussi une dimension relativement activante lorsque l'on se met à explorer les "creux du discours" (Fossion et al., 2018). En outre, elle s'avère potentiellement assez exigeante pour celui qui la conduit. On a vu dans les études de cas qu'il était possible de réagir sur une expression langagière en particulier ou plutôt de synthétiser un ensemble de propos et de les rassembler, au bout d'un moment qui peut être plus ou moins long, dans un recadrage 
global. C'est une distinction que l'on trouve aussi dans la pratique du recadrage en général, certains thérapeutes aimant à rebondir sur une phrase singulière là où d'autres s'efforcent de percevoir un contenu qui traverse un voire plusieurs entretiens. Cette souplesse permet de choisir ses moments et d'utiliser la mise au carré de manière plus ou moins régulière dans le processus d'accompagnement.

Par ailleurs, nous avons, au cours de notre pratique clinique, testé quatre modalités d'usage de la mise au carré. En effet, on peut croiser deux critères :

- la mise au carré peut être pratiquée de manière explicite ou implicite, autrement dit en annonçant à la personne que l'on procède à une combinaison ou en s'en servant de manière intérieure pour faire avancer sa réflexion;

- la mise au carré peut être pratiquée de manière écrite (en représentant concrètement sur une feuille le carré auquel on pense) ou mentale.

Pour faire une dernière illustration de la démarche, c'est par mise au carré que nous allons la présenter.

Ceci donne donc quatre configurations:

\begin{tabular}{|l|l|l|}
\hline & Écrite & Mentale \\
\hline Explicite & $\begin{array}{l}\text { L'intervenant montre à la personne } \\
\text { le tableau de mise au carré et en } \\
\text { discute avec elle }\end{array}$ & $\begin{array}{l}\text { L'intervenant explique à la personne accom- } \\
\text { pagnée de quelle manière elle procède à un } \\
\text { réaménagement de ses propos, sans pour } \\
\text { autant l'écrire }\end{array}$ \\
\hline Implicite & $\begin{array}{l}\text { L'intervenant prend note de la mise } \\
\text { au carré sans pour autant le montrer } \\
\text { à la personne accompagnée }\end{array}$ & $\begin{array}{l}\text { L'intervenant garde en tête sa mise au carré } \\
\text { et conduit l'entretien en fonction de celle-ci }\end{array}$ \\
\hline
\end{tabular}

Tableau 13. Modalités de mobilisation de la mise au carré

Il faut également signaler que thérapeute et patient peuvent être partenaires des choix de mise au carré. Certes, le thérapeute va mobiliser ses compétences professionnelles au service de la démarche, mais le patient est également à même de juger des mises au carré les plus pertinentes à développer par rapport à son histoire. En effet, comme le disent Doutrelugne et ses collaborateurs (2019), le patient est comme un conteur d'histoire, capable de définir lui-même l'orientation de l'intervention, ses objectifs et les moyens d'y parvenir. Le choix des mises au carré peut alors donner lieu à d'intéressantes négociations et alliances entre les partenaires de la relation thérapeutique.

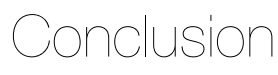

Aider à voir différemment, se placer d'un autre point de vue, prendre en compte d'autres facteurs (Bandler et Grinder, 2014), voilà autant de manières de définir un recadrage. Mais trop souvent, on s'en remet au génie, à l'intuition ou à l'expérience du thérapeute l'accompagnant pour dénicher par quelle voie entrer pour en trouver le contenu. La mise au carré offre une voie d'accès qui va à la fois guider l'attention du professionnel vers des liaisons et lui donner la méthode pour en proposer d'autres. 
Si, par la forme de systématisme qu'elle offre, la technique est de nature à stimuler la créativité, il restera à choisir sur quelle(s) association(s) agir. Le discours d'un patient offre en effet une multitude de mises au carré possibles. Toute la question sera donc de choisir celles qui seront les plus porteuses à un moment donné de la thérapie.

Au final, la mise au carré constitue donc une technique au service du processus thérapeutique qui, sans se substituer à la démarche et aux compétences de celles et ceux qui l'utilisent, offre des ouvertures pour penser et agir autrement.

\author{
Correspondance \\ Daniel Faulx \\ Daniel.Faulx@uliege.be \\ Coryse Moncarey \\ coryse.moncarey@uclouvain.be
}

\title{
Bibliographie
}

Bandler R. et Grinder J., Le Recadrage, Paris, InterÉditions, 2014.

Beaumont A., "De l'autre côté du miroir: quand une thérapeute devient cliente", 2016, www.paradoxes.asso.fr/2016/10/de-l'autre-cote-du-miroir-quand-une-therapeute-devient-cliente.

Benoît D., "Influence, persuasion, manipulation, les techniques thérapeutiques de Palo Alto et la communication au quotidien", in C. Le Bœuf (dir.), Rencontre de Paul Watzlawick, Paris, Dunod, 1999, p. 95-132.

Benoît J.-C., Malarewicz J.-A., Beaujean J., Colas Y. et Kannas S., Dictionnaire clinique des thérapies familiales systémiques, Paris, ESF, 1988.

Betbèze J., Des outils en psychothérapie. Les thérapies orientées sur la solution, 2002, www.c-r-a-a.info/images/colloques/Les_outils_en_psychotherapie_Betbeze_2002.pdf.

Curonici C. et McCulloch P., "L'approche systémique en milieu scolaire: réflexions 20 ans après ", Thérapie familiale, 2004 , vol. $25, \mathrm{n}^{\circ} 3$, p. 381-405.

D’Abate D., "Approche orientée vers les solutions et approche narrative familiale auprès des familles à haut niveau de conflit: stratégies et techniques pour la coordination parentale et le coaching coparental ", Journal of Child Custody, 2016, vol. 13, n 4, p. 269288 (version traduite par Luc Lajeunesse).

De Shazer S. et Dolan Y., More than Miracles. The State of Art of Solution-Focused Brief Therapy, New York, Haworth, 2007.

Doutrelugne Y., Cottencin O., Betbèze J., Barrois I. et Likaj V., Thérapies brèves plurielles: principes et outils pratiques, Elsevier, 2019.

Faulx D. «Le recadrage dans les situations conflictuelles. Description et mise en perspective de l'approche de Michel Monroy", Thérapie familiale, 2006, vol. 27, nº 4, p. 377-397.

Faulx D. et Danse C., Apprendre à penser autrement, Paris, Enrick B., 2017.

Fossion G., Jamin V. et Faulx D., «Investiguer les creux du discours par la technique d'entretien de la mise au carré", Recherches qualitatives, 2018, vol. 37, $\mathrm{n}^{\circ}$ 1, p. 21-56.

White M. et Epsen D. Narrative means to therapeutic ends. Norton, NY, 1992.

Goldbeter-Merinfeld E., "Outils thérapeutiques pour l'approche systémique. Introduction", Cahiers critiques de thérapie familiale et de pratiques de réseaux, 2012, vol. 1, no 48, p. 5-11. 
Haley J., Un thérapeute hors du commun: Milton H. Erickson, Paris, Desclée de Brouwer, 1995. Haley J., Stratégies de la psychothérapie, Toulouse, Érès, 1997.

Hanot M., "Techniques systémiques d'entretien pour travailleur social", Thérapie familiale, 2006, vol. 27, $\mathrm{n}^{\circ} 1$, p. $75-89$.

Isebaert L. et Cabié M.-C., Pour une thérapie brève, Toulouse, Érès, 2015.

Janne P. et Dessoy E., "Intérêt et place du "recadrage" en thérapie familiale: une relecture du concept initiée par un sonnet de Charles Baudelaire", Thérapie familiale, 1999, vol. $20, \mathrm{n}^{\circ} 3$, p. 275-285.

Kourilsky-Belliard F., Du désir au plaisir de changer. Comprendre et provoquer le changement, Paris, Dunod, 1995.

Malarewicz J.-A., Systémique et entreprise, Paris, Village mondial, 2000.

Marsan C., "Le changement de paradigme», in M. Christine (dir.), Réussir le changement. Comment sortir des blocages individuels et collectifs?, Louvain-la-Neuve, De Boeck Supérieur, 2008, p. 43-74.

Miller G. et de Shazer S., "Avez-vous entendu la dernière à propos de...? La thérapie brève centrée sur les solutions en tant que rumeur", Thérapie familiale, 2001, vol. $22, \mathrm{n}^{\circ} 3$, p. 289-308.

Morgan A., Qu'est-ce que l'approche narrative?, Paris, InterÉditions, 2019.

Mori S. et Rouan G. Les thérapies narratives. Bruxelles, De Boeck, 2011.

Nardone G. et Watzlawick P., L'Art du changement. Thérapie stratégique et hypnothérapie sans transe, Paris, L'Esprit du temps, PUF, 1993.

Panichelli C., "Le mécanisme de défense de l'humour: un outil pour le recadrage", Cahiers critiques de thérapie familiale et de pratiques de réseaux, 2007, vol. 2, nº 39, p. 39-56.

Paugam S. "L'exclusion, état des recherches", Sciences Humaines, 13, 12-14, 1996.

Paul M. "Accompagnement ", Recherche et Formation, 62, 91-108, 2009.

Petit S., "Les effets de la "question miracle" dans les textes de patients alcoolodépendants", Psychotropes, 2005, vol. 11, n 3-4, p. 209-225.

Piret A., Nizet J. et Bourgeois E., L'Analyse structurale. Une méthode d'analyse de contenu pour les sciences humaines, Louvain-la-Neuve, De Boeck, 1996.

Robin-Quach P., "Connaître les représentations du patient pour optimiser le projet éducatif ", Recherche en soins infirmiers, 2009, vol. 3, $\mathrm{n}^{\circ} 98$, p. 36-68.

Sprocq-Demarcq N. et Rey Y., "Les objets flottants en formation systémique: contribution au développement personnel et professionnel du futur thérapeute", Cahiers critiques de thérapie familiale et de pratiques de réseaux, 2008, vol. 2, nº 41, p. 69-80.

Wajeman M., "Préface », in S. Minuchin, Familles en thérapie, Toulouse, Érès, 2005, p. 11-16.

Watzlawick P., The Situation Is Hopeless but not Serious. The Pursuit of Unhappiness, New York, Norton, 1993.

Wittezaele J.-J. et García T., À la recherche de l'école de Palo Alto, Paris, Seuil, 1998.

\section{Abstract}

Squaring Language and Thought: A Technique to Support the Reframing Process in Systemic Intervention - Reframing is a central approach in systemic therapy and especially in narrative and solution-oriented therapies. In this article, we present a special technique called squaring. We envisage its deployment to support the reframing process in the context of systemic intervention. Based on the identification of "links", squaring allows existing associations to be visualized in speech and to explore those that are latent but not expressed by the person. It aims for the deployment of potential new associations to support the client's development. This article presents a case study to illustrate the approach. The implications for therapeutic practice are discussed at the end of the article. 


\section{Resumen}

La puesta en cuadro del lenguaje y del pensamiento: una tecnica al servicio del reencuadre en intervencion systémica - El reencuadre constituye una accion central de la terapia sistémica particularmente en las terapias narativas y orientadas a las soluciones. En este articulo, presentamos una tecnica original llamada "Puesta en cuadro". La utilizamos al servicio del reencuadre en un contexto de intervencion sistemica. La Puesta en cuadro permite visualizar las asociaciones existentes en un discurso y explorar cuales estan germinando pero sin ser expresadas por la persona. Esta tecnica sirve para desplegar nuevas asociaciones potenciales al servicio de la evolucion del cliente. El articulo présenta un estudio de caso que viene a ilustrar el planteamiento. Las implicaciones para la practica terapeutica estan discutidas al final del articulo. 